\title{
Total Body Irradiation and Risk of Diabetes Mellitus; A Meta-Analysis
}

\author{
Kittika Poonsombudlert*, Nath Limpruttidham
}

\begin{abstract}
Objective: Hematopoietic stem cell transplant (HSCT) has recently emerged as a cure for previously "incurable" diseases and is being explored and attempted in many other fields including congenital and acquired non-malignant diseases. However, the long-term side effect associated with HSCT especially Total Body Irradiation (TBI) is still understudied. Therefore, we attempted to establish association between TBI and risk of developing Diabetes Mellitus (DM) or impaired glucose metabolism (IGM). Methods: We searched for titles of articles in MEDLINE (PubMed), EMBASE, and Cochrane library in August 2018 that evaluated the association between TBI in the setting of HSCT and DM or IGM. We conducted a random effect meta-analysis of 11 studies involving a total of 13,191 participants and reported the pooled MD (mean difference) for the development of DM/IGM after TBI as part of the conditioning regimen for HSCT. Results: We found a significant increase in the risk of developing DM/IGM after TBI is used as part of the conditioning regimen compared to other types of conditioning regimen with the pooled MD being $5.42,95 \%$ Confidence Interval (CI) $2.51-11.71$, I2 $=92.4 \%$. Conclusion: TBI as a conditioning regimen in the setting of HSCT significantly increases the risk of developing DM/IGM. Therefore, we recommend close monitoring and screening for diabetes mellitus in patients who underwent TBI before HSCT.
\end{abstract}

Keywords: Total body irradiation- hematopoietic stem cell- transplant and diabetes mellitus

Asian Pac J Cancer Prev, 20 (3), 885-891

\section{Introduction}

Hematopoietic stem cell transplant (HSCT) has recently emerged as a cure for previously "incurable" diseases. Its first application was in 1957 for a leukemia patient who received a transfer of stem cell from the healthy twin conferring a "cure" state which was previously not achieved with traditional chemotherapy. (Pidala et al., 2011; Henig and Zuckerman, 2014) Since the breakthrough with the experiment, the horizon of hematopoietic stem cell transplant has expanded to involve other sources of stem cells including cells from unrelated donors or cord blood stem cell stored in the international banks (Kondo et al., 2003; Stacey and Hunt, 2006; Lin et al., 2013) Even now, newer sources such as mesenchymal tissue or other differentiated cellular lineages (Sordi and Piemonti, 2011; Keating, 2012) are also being investigated for cure of various congenital, autoimmune and malignant diseases. It is proper to say that, the potential for application is limitless (Kondo et al., 2003; Stacey and Hunt, 2006; Girlovanu et al., 2015).

However, on the same expanding frontier, the longterm side effects of transplant and accommodating treatment modalities are still understudied. It is estimated that over 8,000 HSCT was performed in the US in 2017 and the number continues to grow for both autologous as well as allogeneic HSCT (D'Souza et al., 2017). From such an astounding number; at the aftermath of the battle, what consequences are the survivors encountering? (Hilgendorf et al., 2015) Previous studies had mentioned the possibilities of developing hypertension, dyslipidemia as well as endocrinologic abnormalities with various risk factors (Baker et al., 2007; Hoffmeister et al., 2010; Wei et al., 2016; Bielorai et al., 2017). Not to mention known co-morbidities from the treatment itself such as graft versus host disease (GVHD) (Socie and Ritz, 2014; Arora et al., 2016) or infectious complications (Nakano et al., 2014; Kumar et al., 2015) which are well known to associate with the immunocompromised state rendered by the treatments (Michael et al., 2013).

Part of the treatment with HSCT is the conditioning regimen which includes a combination of chemotherapy and radiation therapy. Most adverse effects of various chemotherapy regimens including short and long-term complications have been well studied. However, for application of total body irradiation (TBI), the long-term side effect is still to be discovered. The fact that TBI can render the hematopoietic stem cell powerless signifies that other mechanisms are playing a role in other organs and metabolic pathways as well.

We aimed to explore the potential long-term outcome after HSCT and TBI on the development of diabetes 
mellitus (DM) or impaired glucose metabolism (IGM).

\section{Materials and Methods}

\section{Patients and method}

We searched for titles of articles in the MEDLINE (PubMed), EMBASE, and Cochrane library. We performed a search in August 2018 and did not restrict publication dates. The following main search terms were used: hematopoietic stem cell transplant, total/whole body irradiation and diabetes mellitus/impaired glucose metabolism. The full search strategy is detailed in Item $\mathrm{S} 1$ in the Supplementary Material.

All published nonrandomized trials that evaluated the association between TBI in the setting of HSCT and DM or IGM were included. Observational studies-prospective cohort, retrospective cohort and cross-sectional studies-were included. Review articles, case reports, letters, commentaries, abstracts, unpublished studies and studies in languages other than English were not included.

Patient's age was not part of the exclusion criteria as both adult and pediatric patients were included in our analysis. Studies that did not assess the direct association of DM/IGM with TBI or did not have eligible study population were excluded (Chemaitilly et al., 2009; Majhail et al., 2009; Baker et al., 2010; Frisk et al., 2011; Sohn et al., 2011; Bizzarri et al., 2015; Davis et al., 2015; Wei et al., 2015; Wilhelmsson et al., 2015; Wei et al., 2016; Bielorai et al., 2017; Friedman et al., 2017).

The primary outcome was the diagnosis of DM/ IGM, which was measured by standardized blood tests recommended by American Diabetes Association (ADA) (American Diabetes, 2018) or self-report questionnaire given to patients or their primary care physicians. We compared the risks of DM/IGM diagnosis between patients who did and did not receive TBI as part of the conditioning regimen for HSCT.

\section{Data extraction and quality assessment}

Two investigators independently extracted the following data: Authors, publication year, country of origin, study design, baseline patient's characteristics, interventions, and outcomes. Any conflicting opinions on data extraction were resolved by consensus of the investigators.

The Newcastle - Ottawa Quality Assessment Scale (NOS) was used to assess the quality of the nonrandomized studies based on the selection of the study groups, comparability of study groups, and ascertainment of exposure/outcome. Studies with total scores of $>6$ and $<4$ were considered to be of high and low quality, respectively. We excluded any studies that the meta-analysis indicated poor quality. There were no randomized or blinded control trials due to the obvious nature of the intervention.

\section{Statistical methods}

The primary outcome of the study is the pooled mean difference (MD) of the risk of development of DM/ IGM between patients who received TBI compared with patients who did not receive TBI. We used a randomeffects model as included studies are observational study.
We conducted sensitivity analysis and subgroup analysis to explore heterogeneity of the included studies (Higgins et al., 2003). We used Funnel plot (as detailed in Item S2 in the Supplementary Material) and Egger's test to assess for publication bias. All analyses were performed using Stata 13 software.

\section{Results}

\section{Description of included studies}

The initial search yielded 1,388 articles; 1,365 were excluded from title and abstract review as they were obviously not pertaining to our study, did not involve TBI as part of the conditioning regimen, did not evaluate association between TBI and DM/IGM, were not conducted in patients receiving HSCT, conducted in animal models, or were published in languages other than English. A total of 23 articles underwent full-length review. 12 of them were excluded because they did not have a TBI control group or did not have an eligible study population. Finally, we included 11 studies: Five retrospective cohort studies, three prospective cohort studies and two cross-sectional studies. They included 13,191 patients with indications for HSCT, 2,695 who had undergone TBI and 10,266 who had not (controls). Figure S3 In the supplementary material outlined our search methodology and selection process. Table 1 described the characteristics of the extracted studies. The total sample size range was $22-8,599$ patients. The mean age range was 7-44 years. The follow-up duration ranged from 2-30 years. DM/IGM was diagnosed by blood sample per ADA (American Diabetes, 2018) guideline, from self-report or contact with patients' primary care physicians.

\section{Meta-analysis results}

There were 11 studies included in this meta-analysis. Using a random-effects model, the pooled MD of diagnosis of DM/IGM was found to be significantly higher in the TBI group than in the control group (MD $=5.42$, 95\% Confidence Interval (CI) 2.51-11.71). The heterogeneity among studies $\left(\mathrm{I}^{2}\right)$ was $92.4 \%$. We found publication bias from Funnel plot which was not statistically significant from Egger's test $(P=0.466)$. This is likely due to less likelihood of negative studies being selected for publication.

See details for meta-analysis in S4 in Supplementary Material

Subgroup analysis

We conducted a subgroup analysis to assess the effect of age, study design and indication for HSCT on the primary outcome.

First, for age group, we categorized the studies to include only pediatric population and the other for adult patients. Pooled MD among adult group (Baker et al., 2007; Armenian et al., 2012) was 2.09, 95\% CI 0.95-4.61, $\mathrm{I}^{2}=71.7 \%$ while for the pediatric group (Taskinen et al., 2000; Hoffmeister et al., 2004; Neville et al., 2006; Oudin et al., 2011; Michael et al., 2013; Hirabayashi et al., 2014; Oudin et al., 2015; Nakagawa et al., 2018; Shalitin et al., 2018), the pooled MD was 8.43, 95\% CI 5.51-12.91, 


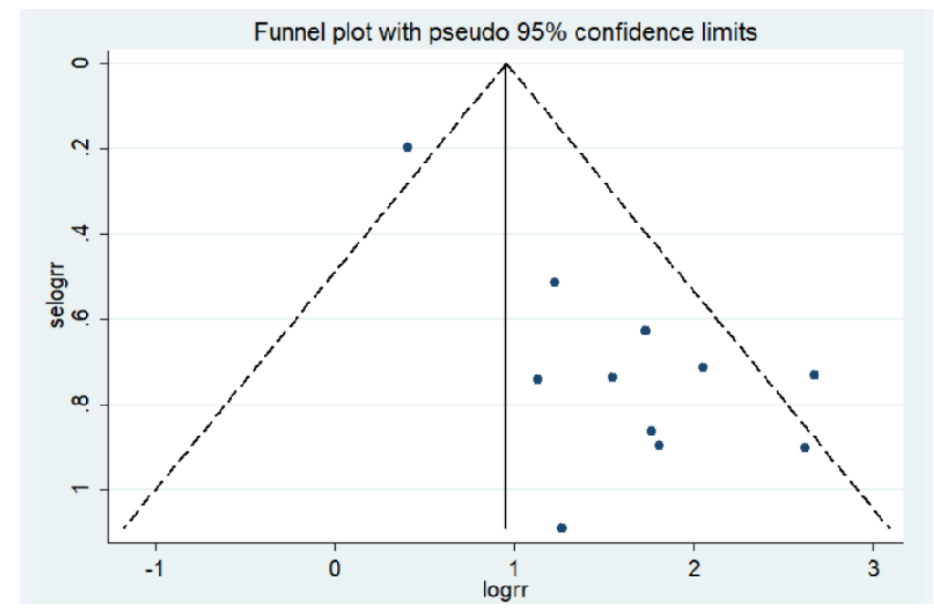

Figure 1. Funnel Plot

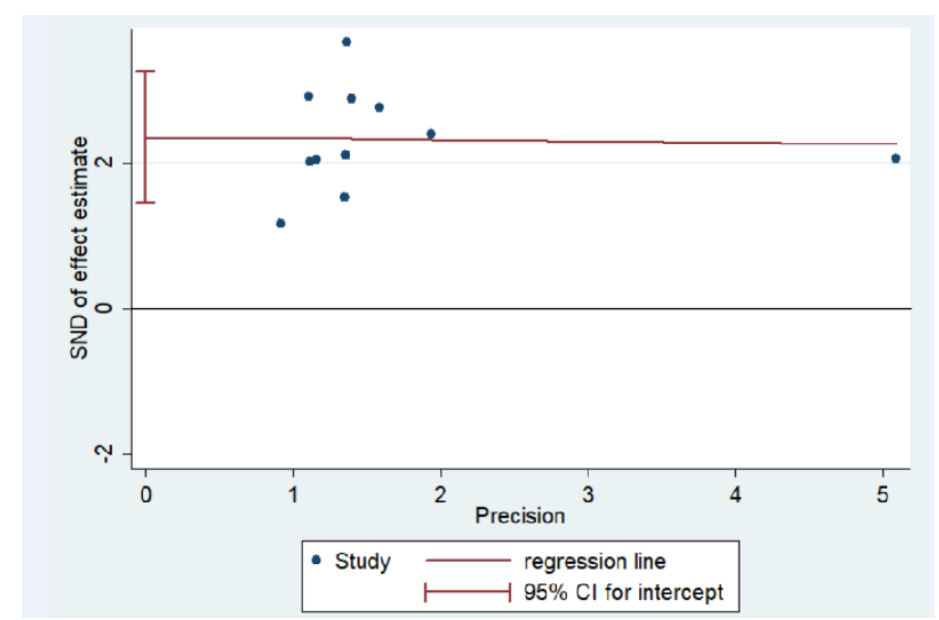

Figure 2. Egger Test

$\mathrm{I}^{2}=44.4 \%$.

In addition, for the study designs, we categorized the studies into prospective, cross-sectional and retrospective studies and found the following pooled MD: $3.21,95 \% \mathrm{CI}$ $1.53-6.73, \mathrm{I}^{2}=71.3 \%$ for retrospective studies (Hoffmeister et al., 2004; Baker et al., 2007; Armenian et al., 2012; Hirabayashi et al., 2014; Shalitin et al., 2018) while for prospective study the pool MD was $12.75,8.77-18.53$, $\mathrm{I}^{2}=16.3 \%$, and $6.76,95 \%$ CI $3.32-13.74, \mathrm{I}^{2}=0 \%$ for cross-sectional studies (Taskinen et al., 2000; Meacham et al., 2010).

Lastly, if we divide the studies with regards to the indication of HSCT. One group evaluates only patients with malignant hematologic $(\mathrm{MaHe})$ condition and the other group for all other conditions. The MaHe group (Hoffmeister et al., 2004; Oudin et al., 2011; Hirabayashi et al., 2014; Oudin et al., 2015) has the pooled MD of 4.98, 95\% CI1.85-13.42, $\mathrm{I}^{2}=0 \%$ and the other studies with pooled MD of $5.55,95 \%$ CI $2.02-15.25, \mathrm{I}^{2}=95.4 \%$ (Taskinen et al., 2000; Neville et al., 2006; Baker et al., 2007; Meacham et al., 2010; Armenian et al., 2012; Nakagawa et al., 2018; Shalitin et al., 2018).

According to this regression meta-analysis, differences in age group and indications for HSCT potentially had effect modification to the association between TBI and the diagnosis of DM/IGM, which could be used to identify high risk population. In addition, we recognized that cross-sectional study design has limitations to accurately diagnose DM/IGM after patients received TBI as the diagnosis is based on a single timeframe rather than a prolonged period of follow-up time in cohort studies. The association remained statistically significant after exclusion of two cross-sectional studies. Therefore, we concluded that a diagnosis of DM/IGM is markedly increased in patients who received TBI as part of the conditioning regimen compared to other conditioning regimens in the setting of HSCT. This observed association was stronger among prospective studies, pediatric population, non-US based studies and studies done exclusively for a malignant hematologic condition.

\section{Discussion}

This meta-analysis investigated the effect of TBI on the development of DM/IGM in patients indicated for HSCT. Our main findings suggested that TBI was significantly associated with an increased risk of the development of DM/IGM compared to those who did not undergo TBI as part of the conditioning regimen for HSCT.

There have been many theories as to why TBI can cause DM/IGM (Chemaitilly et al., 2009). Starting from the fact that TBI; as other types of radiation do is causing inflammation. Most radiation is site-specific, thus limiting the extent of the inflammation to a targeted 


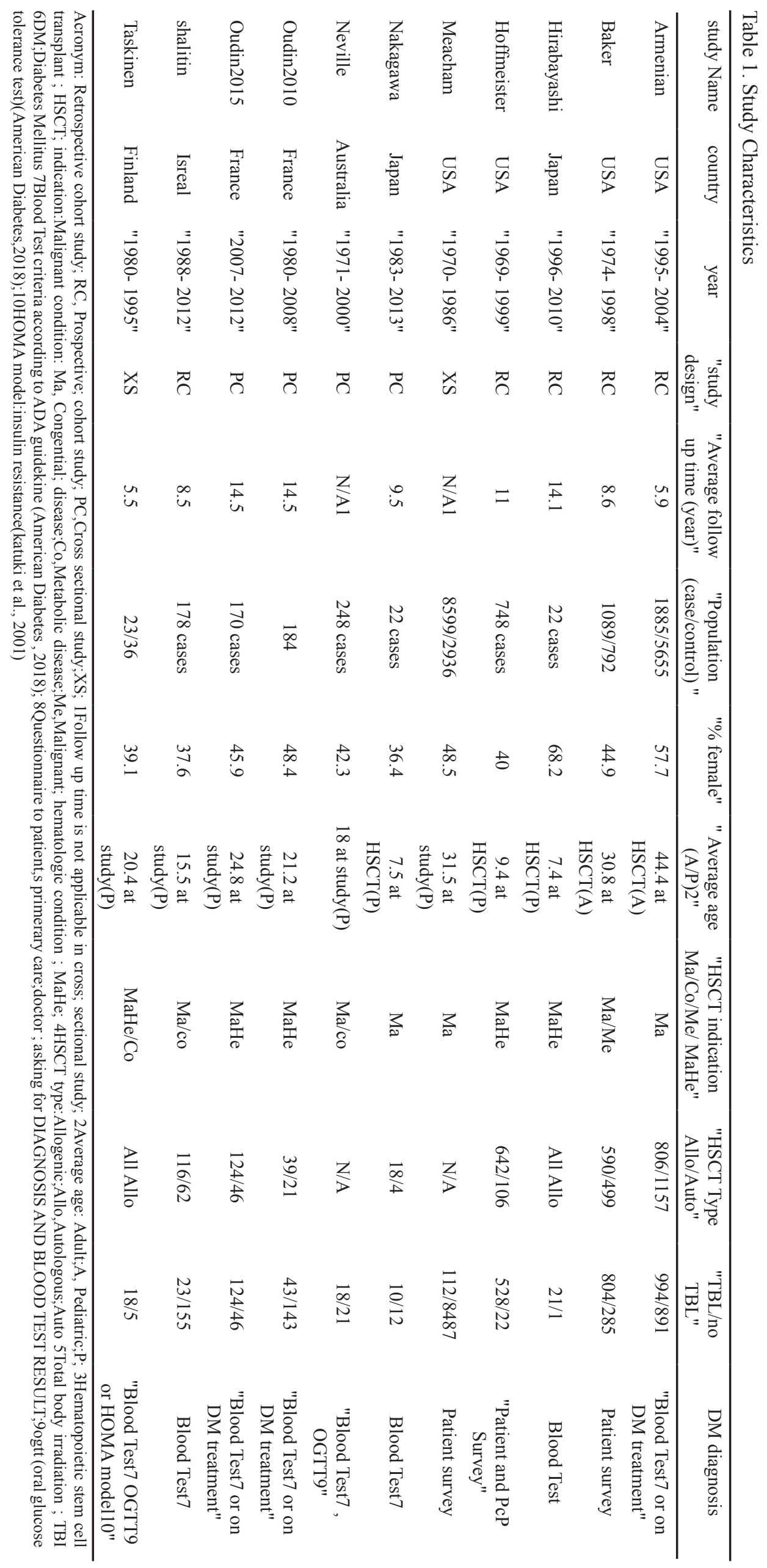


region, however, as the name suggested, TBI; total body irradiation can cause systemic inflammation and interfere with molecular signaling, cascade pathways and more significantly can induce hormonal disturbances. The central result of all the inflammation caused by the TBI is insulin resistance. This is theorized to be from growth hormone deficiency (Martin and Jeanrenaud, 1985; Bakker et al., 2007; Nakagawa et al., 2018) or abnormal adipose tissue redistribution. (Rajendran et al., 2013; Bizzarri et al., 2015) Other more, site-specific issues are from reduced pancreatic beta-cell reserve and an overall decrease in pancreatic volume directly induced by the TBI (Wei et al., 2015; Arora et al., 2016). All of these theories could well explain how TBI can be associated with the development of DM/IGM.

On the contrary, there has long been speculation that various treatments modalities associated with HSCT itself can increase the risk of developing DM/ IGM. (Griffith et al., 2010) including, but not limited to chemotherapy, prolonged use of corticosteroid and various immunosuppressants (Vantyghem et al., 2014; Abou-Mourad et al., 2010; Mostoufi-Moab et al., 2016), which are well known to be associated with truncal obesity and potentially DM/IGM when used for prolonged period of time as well as physical inactivity resulting from prolonging hospitalization (Inoue et al., 2010; Al Tunaiji et al., 2014).

In addition, we found potential publication bias visualized from the Funnel plot, although it did not meet statistical significance in Egger's test. We believed that they were fewer published negative studies, which contributed to publication bias. Although it is generally recognized that small negative studies were difficult to get published in high quality scientific journals, we used general broad search terms as described in supplemental material S1 to include small negative studies as much as we could to minimize publication bias.

There are some limitations to this study. First, only 11 studies met our inclusion criteria, most of which were conducted in the USA, possibly limiting the external validity of this meta-analysis. Secondly, patients of varying age and pre-transplant co-morbidity were included in the study which could be confounding the test result. Thirdly, some studies included patients with known endocrinologic disorders such as hypothyroidism, hypogonadism and growth hormone deficiency which could influence the development of DM/IGM. And lastly, we recognized that there is heterogeneity in the study which is likely due to differences in study designs and background characteristics of the subjects, but according to the regression meta-analysis, we found that change in age, study type, a location of the study and indication for HSCT could potentially explain the heterogeneity.

We believed that there is still more to study regarding an association between TBI, HSCT and development of $\mathrm{DM} / \mathrm{IGM}$ as DM is one of the most debilitating diseases with severe consequences if left untreated (White, 2015). Survivors might not realize that while they are being "cured" of one life-threatening condition; after the treatment, their risk of developing other chronic co-morbidity is not infinitesimal, they should be aware that they are at higher risk of developing DM/IGM and frequent monitoring is warranted (Wei et al., 2016). Our meta-analysis of clinical trials demonstrated that TBI significantly increased the risk of development of DM/ IGM, but this is still an undiscovered field and further research is needed to clarify if the association truly stems directly from TBI or treatment with HSCT as a whole. We suggested that further large, prospective, controlled trials to investigate the long-term outcomes and treatment option for $\mathrm{DM} / \mathrm{IGM}$ in the future as this is a rapidly expanding field of treatment potentials.

\section{Acknowledgements}

Authors certify that they have no affiliations or conflict of interest with or involvement in any organization or entity with any financial or no-financial interest in the subject matter or materials discussed in this manuscript.

\section{References}

Abou-Mourad YR, Lau BC, Barnett MJ, et al (2010). Long-term outcome after allo-SCT: close follow-up on a large cohort treated with myeloablative regimens. Bone Marrow Transplant, 45, 295-302.

Al Tunaiji H, Davis JC, Mackey DC, et al (2014). Population attributable fraction of type 2 diabetes due to physical inactivity in adults: a systematic review. BMC Public Health, 14, 469.

American Diabetes A (2018). 2. Classification and diagnosis of diabetes: Standards of medical care in diabetes-2018. Diabetes Care, 41, 13-27.

Armenian SH, Sun CL, Vase T, et al (2012). Cardiovascular risk factors in hematopoietic cell transplantation survivors: role in development of subsequent cardiovascular disease. Blood, 120, 4505-12.

Arora M, Cutler CS, Jagasia MH, et al (2016). Late acute and chronic Graft-versus-Host disease after allogeneic hematopoietic cell transplantation. Biol Blood Marrow Transplant, 22, 449-55.

Baker KS, Ness KK, Steinberger J, et al (2007). Diabetes, hypertension, and cardiovascular events in survivors of hematopoietic cell transplantation: a report from the bone marrow transplantation survivor study. Blood, 109, 1765-72.

Baker KS, Ness KK, Weisdorf D, et al (2010). Late effects in survivors of acute leukemia treated with hematopoietic cell transplantation: a report from the Bone Marrow Transplant Survivor Study. Leukemia, 24, 2039-47.

Bakker B, Oostdijk W, Geskus RB, et al (2007). Growth hormone (GH) secretion and response to GH therapy after total body irradiation and haematopoietic stem cell transplantation during childhood. Clin Endocrinol (Oxf), 67, 589-97.

Bielorai B, Weintraub Y, Hutt D, et al (2017). The metabolic syndrome and its components in pediatric survivors of allogeneic hematopoietic stem cell transplantation. Clin Transplant, $\mathbf{3 1}$.

Bizzarri C, Pinto RM, Ciccone S, et al (2015). Early and progressive insulin resistance in young, non-obese cancer survivors treated with hematopoietic stem cell transplantation. Pediatr Blood Cancer, 62, 1650-5.

Chemaitilly W, Boulad F, Oeffinger KC, et al (2009). Disorders of glucose homeostasis in young adults treated with total body irradiation during childhood: a pilot study. Bone Marrow Transplant, 44, 339-43.

D'Souza A, Lee S, Zhu X, et al (2017). Current use and trends 
in hematopoietic cell transplantation in the United States. Biol Blood Marrow Transplant, 23, 1417-21.

Davis NL, Stewart CE, Moss AD, et al (2015). Growth hormone deficiency after childhood bone marrow transplantation with total body irradiation: interaction with adiposity and age. Clin Endocrinol (Oxf), 83, 508-17.

Friedman DN, Hilden P, Moskowitz CS, et al (2017). Cardiovascular risk factors in survivors of childhood hematopoietic cell transplantation treated with total body irradiation: A longitudinal analysis. Biol Blood Marrow Transplant, 23, 475-82.

Frisk P, Rossner SM, Norgren S, et al (2011). Glucose metabolism and body composition in young adults treated with TBI during childhood. Bone Marrow Transplant, 46, 1303-8.

Girlovanu M, Susman S, Soritau O, et al (2015). Stem cells - biological update and cell therapy progress. Clujul Med, 88, 265-71.

Griffith ML, Jagasia M, Jagasia SM (2010). Diabetes mellitus after hematopoietic stem cell transplantation. Endocr Pract, 16, 699-706.

Henig I, Zuckerman T (2014). Hematopoietic stem cell transplantation-50 years of evolution and future perspectives. Rambam Maimonides Med J, 5, e0028.

Higgins JP, Thompson SG, Deeks JJ, et al (2003). Measuring inconsistency in meta-analyses. BMJ, 327, 557-60.

Hilgendorf I, Greinix H, Halter JP, et al (2015). Long-term follow-up after allogeneic stem cell transplantation. Dtsch Arztebl Int, 112, 51-8.

Hirabayashi K, Nakazawa Y, Matsuura H, et al (2014). Risk factors for diabetes mellitus and impaired glucose tolerance following allogeneic hematopoietic stem cell transplantation in pediatric patients with hematological malignancies. Int $J$ Hematol, 99, 477-86.

Hoffmeister PA, Hingorani SR, Storer BE, et al (2010). Hypertension in long-term survivors of pediatric hematopoietic cell transplantation. Biol Blood Marrow Transplant, 16, 515-24.

Hoffmeister PA, Storer BE, Sanders JE (2004). Diabetes mellitus in long-term survivors of pediatric hematopoietic cell transplantation. J Pediatr Hematol Oncol, 26, 81-90.

Inoue J, Ono R, Okamura A, et al (2010). The impact of early rehabilitation on the duration of hospitalization in patients after allogeneic hematopoietic stem cell transplantation. Transplant Proc, 42, 2740-4.

Keating A (2012). Mesenchymal stromal cells: new directions. Cell Stem Cell, 10, 709-16.

Kondo M, Wagers AJ, Manz MG, et al (2003). Biology of hematopoietic stem cells and progenitors: implications for clinical application. Annu Rev Immunol, 21, 759-806.

Kumar G, Ahmad S, Taneja A, et al (2015). Severe sepsis in hematopoietic stem cell transplant recipients. Crit Care Med, 43, 411-21.

Lin HT, Otsu M, Nakauchi H (2013). Stem cell therapy: an exercise in patience and prudence. Philos Trans $R$ Soc Lond B Biol Sci, 368, 20110334.

Majhail NS, Challa TR, Mulrooney DA, et al (2009). Hypertension and diabetes mellitus in adult and pediatric survivors of allogeneic hematopoietic cell transplantation. Biol Blood Marrow Transplant, 15, 1100-7.

Martin RJ, Jeanrenaud B (1985). Growth hormone in obesity and diabetes: inappropriate hypothalamic control of secretion. Int J Obes, 9, 99-104.

Meacham LR, Chow EJ, Ness KK, et al (2010). Cardiovascular risk factors in adult survivors of pediatric cancer-a report from the childhood cancer survivor study. Cancer Epidemiol Biomarkers Prev, 19, 170-81.
Michael M, Shimoni A, Nagler A (2013). Recent compounds for immunosuppression and experimental therapies for acute graft-versus-host disease. Isr Med Assoc J, 15, 44-50.

Mostoufi-Moab S, Seidel K, Leisenring WM, et al (2016). Endocrine abnormalities in aging survivors of childhood cancer: A report from the childhood cancer survivor study. $J$ Clin Oncol, 34, 3240-7.

Nakagawa R, Hosokawa-Tsuji A, Aoki Y, et al (2018). Total body irradiation for hematopoietic stem cell transplantation during early childhood is associated with the risk for diabetes mellitus. Endocrine, 61, 76-82.

Nakano N, Kubota A, Tokunaga M, et al (2014). High incidence of CMV infection in adult T-cell leukemia/ lymphoma patients after allogeneic hematopoietic stem cell transplantation. Bone Marrow Transplant, 49, 1548-9.

Neville KA, Cohn RJ, Steinbeck KS, et al (2006). Hyperinsulinemia, impaired glucose tolerance, and diabetes mellitus in survivors of childhood cancer: prevalence and risk factors. J Clin Endocrinol Metab, 91, 4401-7.

Oudin C, Auquier P, Bertrand Y, et al (2015). Metabolic syndrome in adults who received hematopoietic stem cell transplantation for acute childhood leukemia: an LEA study. Bone Marrow Transplant, 50, 1438-44.

Oudin C, Simeoni MC, Sirvent N, et al (2011). Prevalence and risk factors of the metabolic syndrome in adult survivors of childhood leukemia. Blood, 117, 4442-8.

Pidala J, Lee SJ, Quinn G, et al (2011). Variation in management of immune suppression after allogeneic hematopoietic cell transplantation. Biol Blood Marrow Transplant, 17, 1528-36.

Rajendran R, Abu E, Fadl A, et al (2013). Late effects of childhood cancer treatment: severe hypertriglyceridaemia, central obesity, non alcoholic fatty liver disease and diabetes as complications of childhood total body irradiation. Diabet Med, 30, e239-42.

Shalitin S, Pertman L, Yackobovitch-Gavan M, et al (2018). Endocrine and metabolic disturbances in survivors of hematopoietic stem cell transplantation in childhood and adolescence. Horm Res Paediatr, 89, 108-21.

Socie G, Ritz J (2014). Current issues in chronic graft-versus-host disease. Blood, 124, 374-84.

Sohn YB, Kim SJ, Park SW, et al (2011). The metabolic syndrome and body composition in childhood cancer survivors. Korean J Pediatr, 54, 253-9.

Sordi V, Piemonti L (2011). Therapeutic plasticity of stem cells and allograft tolerance. Cytotherapy, 13, 647-60.

Stacey G, Hunt CJ (2006). The UK Stem Cell Bank: a UK government-funded, international resource center for stem cell research. Regen Med, 1, 139-42.

Taskinen M, Saarinen-Pihkala UM, Hovi L, et al (2000). Impaired glucose tolerance and dyslipidaemia as late effects after bone-marrow transplantation in childhood. Lancet, 356, 993-7.

Vantyghem MC, Cornillon J, Decanter C, et al (2014). Management of endocrino-metabolic dysfunctions after allogeneic hematopoietic stem cell transplantation. Orphanet J Rare Dis, 9, 162.

Wei C, Thyagiarajan M, Hunt L, et al (2015). Reduced beta-cell reserve and pancreatic volume in survivors of childhood acute lymphoblastic leukaemia treated with bone marrow transplantation and total body irradiation. Clin Endocrinol (Oxf), 82, 59-67.

Wei C, Unsworth R, Davis N, et al (2016). Survivors of childhood leukaemia treated with haematopoietic stem cell transplantation and total body irradiation should undergo screening for diabetes by oral glucose tolerance tests. Diabet Med, 33, 1347-51.

White NH (2015). Long-term outcomes in youths with diabetes 
mellitus. Pediatr Clin North Am, 62, 889-909.

Wilhelmsson M, Vatanen A, Borgstrom B, et al (2015). Adverse health events and late mortality after pediatric allogeneic hematopoietic SCT-two decades of longitudinal follow-up. Bone Marrow Transplant, 50, 850-7.

\section{(c) (i) (9)}

This work is licensed under a Creative Commons AttributionNon Commercial 4.0 International License. 